\title{
Small Sample Research: Considerations Beyond Statistical Power
}

\author{
Kathleen E. Etz ${ }^{1}$ - Judith A. Arroyo ${ }^{2}$
}

Published online: 19 August 2015

(C) Society for Prevention Research (outside the USA) 2015

\begin{abstract}
Small sample research presents a challenge to current standards of design and analytic approaches and the underlying notions of what constitutes good prevention science. Yet, small sample research is critically important as the research questions posed in small samples often represent serious health concerns in vulnerable and underrepresented populations. This commentary considers the Special Section on small sample research and also highlights additional challenges that arise in small sample research not considered in the Special Section, including generalizability, determining what constitutes knowledge, and ensuring that research designs match community desires. It also points to opportunities afforded by small sample research, such as a focus on and increased understanding of context and the emphasis it may place on alternatives to the randomized clinical trial. The commentary urges the development and adoption of innovative strategies to conduct research with small samples.
\end{abstract}

Small sample research presents a direct challenge to current standards of design and analytic approaches and the underlying notions of what constitutes good prevention science.

The opinions and conclusions here represent those of the authors and do not represent the National Institutes of Health, the National Institute on Drug Abuse, the National Institute on Alcohol Abuse and Alcoholism, or the US Government.

Kathleen E. Etz

ketz@nih.gov

1 National Institute on Drug Abuse, National Institutes of Health, 6001 Executive Blvd., Bethesda, MD 20852, USA

2 National Institute on Alcohol Abuse and Alcoholism, National Institutes of Health, 5635 Fishers Lane, Bethesda, MD 20852, USA
While we can have confidence that our scientific methods have the ability to answer many research questions, we have been limited in our ability to take on research with small samples because we have not developed or adopted the means to support rigorous small sample research. This Special Section identifies some tools that can be used for small sample research. It reminds us that progress in this area will likely require expansion of our ideas of what constitutes rigor in analysis and design strategies that address the unique characteristics and accompanying challenges of small sample research. Advances will also require making room for the adoption of innovative design and statistical analysis approaches. The collection of papers makes a significant contribution to the literature and marks major development in the field.

Innovations in small sample research are particularly critical because the research questions posed in small samples often focus on serious health concerns in vulnerable populations. Individuals most at risk for or afflicted by health disparities (e.g., racial and ethnic minorities) are by definition small in number when compared to the larger, dominant society. The current state of the art in design and statistical analysis in prevention science, which is highly dependent on large samples, has severely handicapped investigation of health disparities in these smaller populations. Unless we develop research techniques suitable for small group design and expand our concepts of what design and analytic strategies provide sufficient scientific rigor, health disparities will continue to lay waste to populations that live in smaller communities or who are difficult to recruit in large numbers. Particularly when considering high-risk, low base rate behaviors such as recurrent binge drinking or chronic drug use, investigators are often limited by small populations in many health disparity groups and by small numbers of potential participants in towns, villages, and rural communities. Even in larger, urban settings, researchers may experience constraints on recruitment such as 
difficulty identifying a sufficiently large sample, distrust of research, lack of transportation or time outside of work hours, or language issues. Until now, small sample sizes and the lack of accepted tools for small sample research have decreased our ability to harness the power of science to research preventive solutions to health disparities. The collection of articles in this Special Section helps to address this by bringing together multiple strategies and demonstrating their strength in addressing research questions with small samples.

Small sample research issues also arise in multi-level, group-based, or community-level intervention research (Trickett et al. 2011). An example of this is a study that uses a media campaign and compares the efficacy of that campaign across communities. In such cases, the unit of analysis is the group, and the limited number of units that can be feasibly involved in a study makes multi-level intervention research inevitably an analysis of small samples. The increasingly recognized importance of intervening in communities at multiple levels (Frohlich and Potvin 2008) and the desire to understand the efficacy and effectiveness of multi-level interventions (Hawe 1994) increase the need to devise strategies for assessing interventions conducted with small samples.

The Special Section makes a major contribution to small sample research, identifying tools that can be used to address small sample design and analytic challenges. The articles here can be grouped into four areas: (1) identification of refinements in statistical applications and measurement that can facilitate analyses with small samples, (2) alternatives to randomized clinical trial (RCT) designs that maintain rigor while maximizing power, (3) use of qualitative and mixed methods, and (4) Bayesian analysis. The Special Section provides a range of alternative strategies to those that are currently employed with larger samples. The first and last papers in the Special Section (Fok et al. 2015; Henry et al. 2015a) examine and elaborate on the contributions of these articles to the field. As this is considered elsewhere, we will focus our comments more on issues that are not already covered but that will be increasingly important as this field moves forward.

One challenge that is not addressed by the papers in this Special Section is the generalizability of small sample research findings, particularly when working with culturally distinct populations. Generalizability poses a different obstacle than those associated with design and analysis, in that it is not related to rigor or the confidence we can have in our conclusions. Rather, it limits our ability to assume the results will apply to populations other than those from whom a sample is drawn and, as such, can limit the application of the work. The need to discover prevention solutions for all people, even if they happen to be members of a small population, begs questions of the value of generalizability and of the importance ascribed to it. Further, existing research raises longstanding important questions about whether knowledge produced under highly controlled conditions can generalize to ethnoculturally diverse communities (Atkins et al. 2006; Beeker et al. 1998; Green and Glasgow 2006). Regardless, the inability to generalize beyond a small population can present a barrier to funding. When grant applications are reviewed, projects that are not seen as widely generalizable often receive poor ratings. Scientists conducting small sample research with culturally distinct groups are frequently stymied by how they can justify their research when it is not generalizable to large segments of the population. In some instances, the question that drives the research is that which limits generalizability. For example, research projects on cultural adaptations of established interventions are often highly specific. An adaptation that might be efficacious in one small sample might not be so in other contexts. This is particularly the case if the adaptation integrates local culture, such as preparing for winter and subsistence activities in Alaska or integrating the horse culture of the Great Plains. Even if local adaptation is not necessary, dissemination research to ascertain the efficacy and/or effectiveness of mainstream, evidence-based interventions when applied to diverse groups will be difficult to conduct if we cannot address concerns about generalizability.

It is not readily apparent how to address issues of generalizability, but it is clear that this will be challenging and will require creativity. One potential strategy is to go beyond questions of intervention efficacy to address additional research questions that have the potential to advance the field more generally. For example, Allen and colleagues' (2014) scientific investigations extended beyond development of a prevention intervention in Alaska Native villages to identification and testing of the underlying prevention processes that were at the core of the culturally specific intervention. This isolation of the key components of the prevention process has the potential to inform and generalize across settings. The development of new statistical tools for small culturally distinct samples might also be helpful in other research contexts. Similarly, the identification of the most potent prevention processes for adaptation also might generalize. As small sample research evolves, we must remain open to how this work has the potential to be highly valuable despite recognizing that not all aspects of it will generalize and also take care to identify what can be applied generally.

While not exclusive to small sample research, additional difficulties that can arise in conducting research in some small, culturally distinct samples are the questions of what constitutes knowledge and how to include alternative forms of knowledge (e.g., indigenous ways of knowing, folk wisdom) in health research (Aikenhead and Ogawa 2007; Gone 2012). For many culturally distinct communities that turn to research to address their health challenges, the need for large samples and methods demanded by mainstream science might be incongruent with local epistemologies and cultural understandings of how the knowledge to inform prevention is generated and standards of evidence are established. Making sense of 
how or whether indigenous knowledge and western scientific approaches can work together is an immense challenge. The Henry, Dymnicki, Mohatt, Kelly, and Allen article in this Special Section recommends combining qualitative and quantitative methods as one way to address this conundrum. However, this strategy is not sufficient to address all of the challenges encountered by those who seek to integrate traditional knowledge into modern scientific inquiry. For culturally distinct groups who value forms of knowledge other than those generated by western science, the research team, including the community members, will need to work together to identify ways to best ensure that culturally valued knowledge is incorporated into the research endeavor. The scientific field will need to make room for approaches that stem from the integration of culturally valued knowledge.

Ensuring that the research design and methods correspond to community needs and desires can present an additional challenge. Investigations conducted with small, culturally distinct groups often use community-based participatory research (CBPR) approaches (Minkler and Wallerstein 2008). True CBPR mandates that community partners be equal participants in every phase of the research, including study design. From an academic researcher's perspective, the primary obstacle for small sample research may be insufficient statistical power to conduct a classic RCT. However, for the small group partner, the primary obstacle may be the RCT design itself. Many communities will not allow a RCT because assignment of some community members to a no-treatment control condition can violate culturally based ethical principles that demand that all participants be treated equally. Particularly in communities experiencing severe health disparities, community members may want every person to receive the active intervention. While the RCT has become the gold standard because it is believed to be the most rigorous test of intervention efficacy, it is clear the RCT does not serve the needs of all communities.

While presenting challenges for current methods, it is important to note that small sample research can also expand our horizons. For example, attempts to truly comprehend culturally distinct groups will lead to a better understanding of the role of context in health outcomes. Current approaches more often attempt to control for extraneous variables rather than work to more accurately model potentially rich contextual variables. This blinds us to cultural differences between and among small groups that might contribute to outcomes and improve health. Analytical strategies that mask these nuances will fail to detect information about risk and resilience factors that could impact intervention. Multi-level intervention research (which we pointed out earlier qualifies as small sample research) that focuses on contextual changes as well as or instead of change in the individual will also inform our understanding of context, elucidating how to effectively intervene to change context to promote health outcomes. Thus, considering how prevailing methods limit our work in small samples can also expose ways that alternative methods may advance our science more broadly by enhancing both our understanding of context and how to intervene in context.

Small sample science requires us to consider alternatives to the RCT, and this consideration introduces additional opportunities. The last paper in this Special Section (Henry et al. 2015b) notes compelling critiques of RCT. Small sample research demands we incorporate alternate strategies that may be superior in some instances regarding their efficiency in their use of available information, in contrast to the classic RCT, and may be more aligned with community desires. Alternative designs for small sample research may offer means to enhance and ensure scientific rigor without depending on RCT design (Srinivasan et al. 2015). It is important to consider what alternative approaches can contribute rather than adhering rigidly to the RCT.

New challenges require innovative solutions. Innovation is the foundation of scientific advances. It is one of only five National Institutes of Health grant review criteria. Despite the value to science of innovation, research grant application reviewers are often skeptical of new strategies and are reluctant to support risk taking in science. As a field, we seem accustomed to the use of certain methods and statistics, generally accepting and rarely questioning if they are the best approach. Yet, it is clear that common methods that work well with large samples are not always appropriate for small samples. Progress will demand that new approaches be well justified and also that the field supports innovation and the testing of alternative approaches. Srinivasan and colleagues (2015) further recommend that it might be necessary to offer training to grant application peer reviewers on innovative small sample research methods, thus ensuring that they are knowledgeable in this area and score grant applications appropriately. Alternative approaches need to be accepted into the repertoire of available design and assessment tools. The articles in this Special Section all highlight such innovation for small sample research.

It would be a failure of science and the imagination if newly discovered or re-discovered (i.e., Bayesian) strategies are not employed to facilitate rigorous assessment of interventions in small samples. It is imperative that the tools of science do not limit our ability to address pressing public health questions. New approaches can be used to address contemporary research questions, including providing solutions to the undue burden of disease that can and often does occur in small populations. It must be the pressing nature of the questions, not the limitations of our methods, that determines what science is undertaken (see also Srinivasan et al. 2015). While small sample research presents a challenge for prevailing scientific approaches, the papers in this Special Section identify ways to move this science forward with rigor. It is imperative that the field accommodates these advances, and continues to be 
innovative in response to the challenge of small sample research, to ensure that science can provide answers for those most in need.

\section{Compliance with Ethical Standards}

Funding No external funding supported this work.

Conflict of Interest The authors declare that they have no conflict of interest.

Ethical Approval This article does not contain any studies with human participants or animals performed by any of the authors.

Informed Consent Because this article is a commentary, informed consent is not applicable.

\section{References}

Aikenhead, G. S., \& Ogawa, M. (2007). Indigenous knowledge and science revisited. Cultural Studies of Science Education, 2, 539-620.

Allen, J., Mohatt, G. V., Fok, C. C. T., Henry, D., Burkett, R., \& People Awakening Project. (2014). A protective factors model for alcohol abuse and suicide prevention among Alaska Native youth. American Journal of Community Psychology, 54, 125-139.

Atkins, M. S., Frazier, S. L., \& Cappella, E. (2006). Hybrid research models: Natural opportunities for examining mental health in context. Clinical Psychology Review, 13, 105-108.

Beeker, C., Guenther-Grey, C., \& Raj, A. (1998). Community empowerment paradigm drift and the primary prevention of HIV/AIDS. Social Science \& Medicine, 46, 831-842.
Fok, Henry, D., Allen, J. (2015). Maybe small is too small a term: Introduction to advancing small sample prevention science. Prevention Science.

Frohlich, K. L., \& Potvin, L. (2008). Transcending the known in public health practice: The inequality paradox: The population approach and vulnerable populations. American Journal of Public Health, 98, 216-221.

Gone, J. P. (2012). Indigenous traditional knowledge and substance abuse treatment outcomes: The problem of efficacy evaluation. American Journal of Drug and Alcohol Abuse, 38, 493-497.

Green, L. W., \& Glasgow, R. E. (2006). Evaluating the relevance, generalization, and applicability of research: Issues in external validation and translation methodology. Evaluation \& the Health Professions, $29,126-153$.

Hawe, P. (1994). Capturing the meaning of "community" in community intervention evaluation: Some contributions from community psychology. Health Promotion International, 9, 199-210.

Henry, D., Dymnicki, A. B., Mohatt, N., Kelly, J. G., \& Allen, J. (2015a). Clustering methods with qualitative data: A mixed methods approach for prevention research with small samples. Prevention Science. doi:10.1007/s11121-015-0561-z.

Henry, D., Fok, C.C.T., Allen, J. (2015). Why small is too small a term: Prevention science for health disparities, culturally distinct groups, and community-level intervention. Prevention Science.

Minkler, M., \& Wallerstein, N. (Eds.). (2008). Community-based participatory research for health: From process to outcomes (2nd ed.). San Francisco: Jossey-Bass.

Srinivasan, S., Moser, R. P., Willis, G., Riley, W., Alexander, M., Berrigan, D., \& Kobrin, S. (2015). Small is essential: Importance of subpopulation research in cancer control. American Journal of Public Health, 105, 371-373.

Trickett, E. J., Beehler, S., Deutsch, C., Green, L. W., Hawe, P., McLeroy, K., Miller, R. L., Rapkin, B. D., Schensul, J. J., Schulz, A. J., \& Trimble, J. E. (2011). Advancing the science of community-level interventions. American Journal of Public Health, 11, 1410-1419. 